\title{
A PROBLEM OF SCHMIDT AND SPITZER
}

\author{
BY J. L. ULLMAN
}

Communicated by A. P. Calderon, June 5, 1967

Introduction. In $\$ 1$ we formulate a question posed in [5] concern. ing the eigenvalues of the sections of a Toeplitz form, and summarize needed results on this subject from [4] and [5]. In $\$ 2$ we prove a theorem which we then apply to answer the question affirmatively.

1.1. Toeplitz forms. Let $\left\{a_{n}\right\}, n=\cdots,-1,0,1, \cdots$ be the Fourier coefficients of a bounded, measurable, complex valued function defined on $[0,2 \pi]$. The Toeplitz form $\left[a_{m-n}\right], m, n=0,1, \cdots$, can be considered as an operator on $\left\{\left[x_{n}\right]\right\}, n=0,1, \cdots$, where $\sum\left|x_{i}\right|^{2}<\infty$. Widom [6] has shown that $\sigma^{+}$, the spectrum of this operator, is connected, answering a question posed in [3].

1.2. Sections of a Toeplitz form. Let $M_{n}$ denote the matrix $\left[a_{m-n}\right]$, $m, n=0,1, \cdots, n$. The eigenvalues of $M_{n}$, namely the zeros of $D_{n}(\lambda)=\operatorname{det}\left[M_{n}-\lambda I_{n}\right]$, are $\lambda_{0, n}, \cdots, \lambda_{n, n}$ and we denote the set by $\sigma_{n}$. Let $B=\left\{\lambda \mid \lambda=\lim \lambda_{n}, \lambda_{n} \in \sigma_{i_{n}}, i_{n} \rightarrow \infty\right\}$. In the Hermitian case, that is when $a_{m}=\bar{a}_{-m}$, there is an extensive theory of the distribution of the $\left\{\lambda_{i, n}\right\}[2]$, and in particular $B=\sigma^{+}$. In the non-Hermitian case the main results are found in [4] and [5] where the added hypothesis $a_{n}=0$ for $|n| \geqq p>0$ is made. We call this the doubly restricted case of Toeplitz sections, since progress has been made in the singly restricted case [1], that is when $a_{n}=0$ for $n \geqq p>0$. This may prove to be an avenue of access to the unrestricted case.

1.3. A question on doubly-restricted Toeplitz sections. Let $f(z)$ $=\sum_{-k}^{h} a_{z} z^{\nu}$, where $a_{-k} \neq 0$ and $-k \leqq-1$. Let $\alpha_{i}(\lambda), i=1, \cdots, k+h$, be the moduli of the zeros of $z^{k}(f(z)-\lambda)$, with repetitions for multiple zeros and indexed so that $\alpha_{i}(\lambda) \leqq \alpha_{i+1}(\lambda), i=1, \cdots, h+k-1$. If $C=\left\{\lambda \mid \alpha_{k}(\lambda)=\alpha_{k+1}(\lambda)\right\}$, then it is shown in [5] that $C=B$. The question is raised whether $C$ is connected. This will be answered in the affirmative. We note the parallel between this question and the one mentioned in 1.1.

1.4. Some needed results. In simplifying and extending the results of [5], Hirschman has made explicit the structure of $C$, which is implicit in [5].

Lemma 1. $C$ can be represented as a finite union of closed analytic arcs, where either distinct arcs are disjoint, or, if not, their intersection consists of one or both common end points. None of the arcs are degenerate, i.e., points. 
We cite further results from [4].

LEMMA 2. There is a function $G(\lambda)$, single valued and analytic in $\tilde{C}$, the complement of $C$ in the finite plane, and nonvanishing there, such that $\lim _{n \rightarrow \infty} D_{n}(\lambda) / D_{n-1}(\lambda)=G(\lambda)$ for $\lambda \in \widetilde{C}$.

Let $\alpha_{n}$ be the measure defined on the Borel sets of the plane by the formula $\alpha_{n}(E)=\left(\sum_{\lambda_{i, n} \in E} 1\right) /(n+1)$.

LEMma 3. If $N$ is any neighborhood of $C$, then the support of $\alpha_{n}$ is contained in $N$, provided $n$ is sufficiently large.

Lemma 4. The measures $\alpha_{n}$ converge weakly to a measure $\alpha$ with support $C$.

2.1. THEOREM. (a) Let $\mu$ be a unit measure with compact carrier $K$ having finitely many components, none of which is a point. (b) Let $U(z)=\int \log |z-t| d \mu$. Let $\widetilde{K}=\cup O_{i}$, where $O_{i}$ are disjoint, connected open sets. Let $U_{j}(z)$ be the restriction of $U(z)$ to $O_{j}$. These functions are harmonic and we let $\tilde{U}_{j}(z)$ be a harmonic function, possibly multiple valued, conjugate to $U_{j}(z)$. If $\phi_{j}(z)=\exp \left(U_{j}(z)+i \widetilde{U}_{j}(z)\right)$ are single valued, analytic functions in $O_{j}, j=1,2, \cdots$, then (c) $K$ is connected.

\subsection{Proof of the Theorem.}

Lemma 5. If $K_{1}, \cdots, K_{n}$ are $n$ pair-wise disjoint, bounded continua, none of which is a point, then there is an analytic Jordan curve containing precisely one of them and not intersecting any $K_{i}$.

Proof of Lemma 5. There must be one continuum, say $K_{1}$, not containing any of the others in any bounded component of its complement. The unbounded component of its complement is a Dirichlet domain and has a Green's function $G(z)$. If $G(z) \geqq \lambda>0$ for $z \in \cup_{i=2}^{n} K_{i}$, then $G(z)=\lambda / 2$ defines an analytic Jordon curve with the desired properties.

Lemma 6. If $\mu$ is a finite measure with compact support $K$, and if $\mathrm{\Gamma}$ is an analytic Jordon curve in $\tilde{K}$ and $K_{1}$ the portion of $K$ inside $\Gamma$, then

$$
\mu\left(K_{1}\right)=\frac{1}{2 \pi} \int_{\Gamma} \frac{\partial}{\partial n} U(z) d s=\frac{1}{2 \pi} \int_{\Gamma} \frac{\partial}{\partial s} U(z) d s=\frac{1}{2 \pi} \Delta_{\Gamma} \tilde{U}(z),
$$

where $\partial / \partial n$ denotes exterior normal derivative, $\partial / \partial s$ denotes counterclockwise tangential derivative and $\Delta_{\Gamma} \tilde{U}(z)$ is the change in a determination of $\tilde{U}(z)$ when it is continued continuously in a counterclockwise direction around $\mathrm{\Gamma}$, starting at an arbitrary point. 
Proof of Lemma 6. The first equality follows from the Gauss theorem, the Cauchy-Riemann equations yield the next equality, and the third follows from integrating a derivative.

Proof of Theorem Completed. If $K$ is not connected, by Lemma 5 there is an analytic Jordon curve $\Gamma$ in $\tilde{K}$ containing precisely one component of $K$, say $K_{1}$. Let $\Gamma$ be in $O_{1}$. Since $0<\mu\left(K_{i}\right)$ and $\sum_{i=1}^{n} \mu\left(K_{i}\right)=1$, it follows that $0<\mu\left(K_{1}\right)<1$. Since $\Delta_{\Gamma} \phi_{1}(z)$ $=\exp \left(\Delta_{\Gamma} U_{1}(z)+i \Delta_{\Gamma} \tilde{U}_{1}(z)\right)$ it follows from Lemma 6 that $\phi_{1}(z)$ is not single valued in $O_{1}$. This contradiction proves the theorem.

2.3. An Application. It follows from Lemma 2 that

$$
\lim _{n \rightarrow \infty}\left(\left|D_{n}(\lambda)\right|\right)^{1 / n}=|G(\lambda)|, \quad \lambda \in \dot{C} .
$$

This can be written as

$$
\lim _{n \rightarrow \infty} \exp \int \log |\lambda-t| d \alpha_{n}=|G(\lambda)| .
$$

Then using Lemmas 3 and 4 we obtain

$$
U(\lambda)=\int \log |\lambda-t| d \alpha=\log |G(\lambda)|, \quad \lambda \in \tilde{C} .
$$

Now by Lemmas 1 and 4 we see that hypothesis (a) of the theorem is satisfied by the measure $\alpha$. We also see that

$$
U_{j}(\lambda)+i \tilde{U}_{j}(\lambda)=\log G(\lambda)+i C_{j}, \quad \lambda \in O_{j},
$$

so that $\phi_{j}(\lambda)$ is single valued, satisfying hypothesis (b) of the theorem. Hence the connectedness of $C$ follows from the conclusion of the theorem.

\section{REFERENCES}

1. G. Baxter and P. Schmidt, Determinants of a certain class of non-Hermitian Toeplitz matrices, Math. Scand. 9 (1961), 122-128.

2. U. Grenander and G. Szegö, Toeplitz forms and their applications, Univ. of California Press, Berkeley, Calif., 1958.

3. P. R. Halmos, "A glimpse into Hilbert space" in $A$ survey of modern mathematics, Wiley, New York, 1963.

4. I. I. Hirschman, Jr., The spectra of certain Toeplitz matrices, Ill. J. Math. 11 (1967), 145-159.

5. P. Schmidt and F. Spitzer, The Toeplitz matrices of an arbitrary Laurent polynomial, Math. Scand. 8 (1960), 15-38.

6. H. Widom, On the spectrum of a Toeplitz operator, Pacific J. Math. 14 (1964), 365-375.

University of Michigan 